\title{
A fundamental solution based FE model for thermal analysis of nanocomposites
}

\author{
H. Wang ${ }^{1,2}$ \& Q. H. Qin ${ }^{2}$ \\ ${ }^{I}$ Institute of Scientific and Engineering Computation, \\ Henan University of Technology, China \\ ${ }^{2}$ School of Engineering, Australian National University, Australia
}

\begin{abstract}
This paper presents a fundamental solution (FS) based finite element (FE) formulation for analyzing the axisymmetric thermal behavior of composites enhanced with carbon nanofibers (CNFs) or carbon nanotubes (CNTs), which are modeled by a cylindrical representative volume element (RVE). The proposed approach utilizes the axisymmetric FS to construct an intra-element approximate field within the element and describes the element boundary field using conventional shape functions. A new hybrid variational functional is developed to establish a linkage between the independent intra-element field and the element boundary fields and generate the final force-displacement equations. Several numerical examples are considered to assess the efficiency and accuracy of the proposed model. The results show that the radius of the nanofiller and the thickness of the interface have little effect on thermal conductivity of the composites, whereas the length of the nanofiller and the material parameters of the interface play an important role in the effective thermal conductivity of the composites.
\end{abstract}

Keywords: nanocomposites, fundamental solution, hybrid FEM, thermal conductivity, cylindrical representative volume element.

\section{Introduction}

Over the past decades, nanomaterials have been used increasingly as ideal additives to polymers, due to their excellent thermal, mechanical, and electrical properties over conventional fillers like carbon fiber and glass fiber [1]. Here we 
direct our attention to the thermal analysis of $\mathrm{CNF} / \mathrm{CNT}$ reinforced composites to simulate the heat dissipation in them, rather than their mechanical simulation.

Currently, the effective thermal properties of nanocomposites have been studied by utilizing experimental techniques [2], molecular scale simulation (i.e. a molecular dynamics (MD) approach [3]), and continuum mechanical models [4]. Among studies based on continuum models, very little numerical work has been carried out to predict the thermal property of nanocomposites. Nishimura and Liu [5] analyzed the thermal behavior of nanocomposites embedded with rigid CNT inclusions by the boundary integral equation (BIE) method with the help of fast multipole expansion. Zhang et al. [6] developed a hybrid boundary node method (BNM) for a multi-domain model, in which both polymer matrix and CNT were modeled separately and the necessary continuity condition on the interface of matrix and CNT was required, and a simplified single-domain model with the assumption that the surface temperature of the CNT filler keeps constant [7]. Singh et al. [8] studied the thermal behavior of nanocomposites by the element-free Galerkin method (EFGM). Furthermore, interphases formed due to chemical reactions between the $\mathrm{CNT} / \mathrm{CNF}$ and matrix, or the use of protective coatings on the filler during manufacturing, can significantly affect the overall thermal properties of composites. Singh et al. [9] applied the EFGM to analyze interface effects by adjusting interface thickness and interface material parameters.

In the present work, a new hybrid FE formulation called HFS-FEM is developed to analyze the size effect of CNT/CNF and the interface effect on the overall thermal conductivity of nanocomposites. As an extension of our previous works [10-12], in the current computational model, a nanoscale axisymmetric cylindrical representative volume element (RVE) containing a single centered effective solid nanofiber is considered. The FS of axisymmetric problems are employed to construct the intra-element temperature field and an independent frame field along the element boundary is defined using conventional shape functions. Then, a new hybrid variational functional is presented to link two assumed fields and to generate the final solving system including boundary integrals only. During the solution process, no any hypersingular integral is involved, though the FS is used, and the element shape can theoretically be arbitrary.

The paper begins with a basic description of axisymmetric heat conduction problems in a nanoscale cylindrical RVE in Section 2. Then, the derivation of the proposed approach is described in Section 3 to provide full insight into the hybrid FE model. The effects of the dimensions of the nanofiller, including its length and diameter, as well as the interface between the nanofiller and matrix are analyzed numerically in Section 4 and some concluding remarks are presented in Section 5. 


\section{Mathematical model for axisymmetric problems}

\subsection{General mathematical model of axisymmetric heat conduction}

Without loss of generality, consider heat conduction in an orthotropic anisotropic homogeneous medium. In a cylindrical coordinate system $(r, \theta, z)$ the governing equation can be expressed as

$$
\frac{\partial}{\partial r}\left(k_{r} \frac{\partial u}{\partial r}\right)+k_{r} \frac{1}{r} \frac{\partial u}{\partial r}+\frac{1}{r^{2}} \frac{\partial}{\partial \theta}\left(k_{\theta} \frac{\partial u}{\partial \theta}\right)+\frac{\partial}{\partial z}\left(k_{z} \frac{\partial u}{\partial z}\right)=0, \quad \forall \mathbf{x} \in \Omega .
$$

and the heat flux components related to the temperature gradient are modeled by Fourier's law given in the following formulation

$$
q_{r}=-k_{r} \frac{\partial T}{\partial r}, q_{\theta}=-\frac{k_{\theta}}{r} \frac{\partial T}{\partial \theta}, q_{z}=-k_{z} \frac{\partial T}{\partial z} .
$$

where $k_{r}, k_{\theta}$ and $k_{z}$ denote the thermal conductivity along the $r, \theta$ and $z$ directions, respectively; $u$ is the sought temperature field in terms of spatial cylindrical coordinate $\mathbf{x}=(r, \theta, z)$.

When there is an axisymmetric geometry and boundary conditions about a reference axis, say $z$ axis, that is, all quantities such as temperature and heat flux components are independent of the circumferential direction ( $\theta$ direction), the governing equation (1) for three-dimensional cases reduces to

$$
\frac{\partial}{\partial r}\left(k_{r} \frac{\partial u}{\partial r}\right)+k_{r} \frac{1}{r} \frac{\partial u}{\partial r}+\frac{\partial}{\partial z}\left(k_{z} \frac{\partial u}{\partial z}\right)=0, \quad \forall \mathbf{x} \in \Omega .
$$

To keep the system solvable, temperature and heat flux boundary conditions should be added

$$
u=\bar{u}, \text { on } \Gamma_{u} ; q=\bar{q}, \quad \text { on } \Gamma_{q} .
$$

where $q=-k_{i} u_{, i} n_{i}$ is the boundary normal heat flux, where in the axisymmetric model the subscript $i$ represents the coordinates $r(i=1)$ and $z(i=2)$, respectively. $n_{i}$ are the direction cosines of the unit outward normal vector $\mathbf{n}$ to the boundary $\Gamma=\Gamma_{u} \cup \Gamma_{q}$ of the domain of interest, and $\bar{u}$ and $\bar{q}$ are specified functions on the corresponding boundaries, respectively. For convenience, the space derivatives are indicated above by a comma, i.e. $u_{, r}=\partial u / \partial r$.

For multi-material problems, the interface continuity conditions for temperature and heat flux should be complemented, i.e. for the subdomains $\Omega_{i}$ and $\Omega_{j}$ with different thermal properties $k_{i}$ and $k_{j}$, respectively. On the interface $\Omega_{i} \cap \Omega_{j}$ we have continuity of temperature and reciprocity of heat flux

$$
u_{i}=u_{j}, \quad q_{i}+q_{j}=0 .
$$




\subsection{Nanoscale cylindrical representative volume element (RVE)}

In the present work, a nanoscale cylindrical RVE (see Fig. 1a) is used to evaluate the effective thermal conductivity of the nanocomposites. The nanofiller is placed symmetrically at the center of the cylindrical RVE such that the axis of the RVE of interest coincides with the axis of the nanofiller. Assume that there is perfect bonding on the interface between the nanofiber and the interphase, and the interphase and the surrounding matrix. The top and bottom surfaces of the RVE are maintained at two different constant temperatures $u_{1}$ and $u_{0}$, respectively, and the outer surface is kept insulated. In Fig. 1, the cylindrical RVE has length $L$ and radius $R_{0}$, and the nanofiber has length $l$ and radius $r_{0}$.

Because the specified geometry and boundary conditions are axisymmetric, a simplified 2D axisymmetric computational model is chosen as a typical rotating plane shown in Fig. 1b, which will be solved by means of the hybrid FE model developed in the present work.
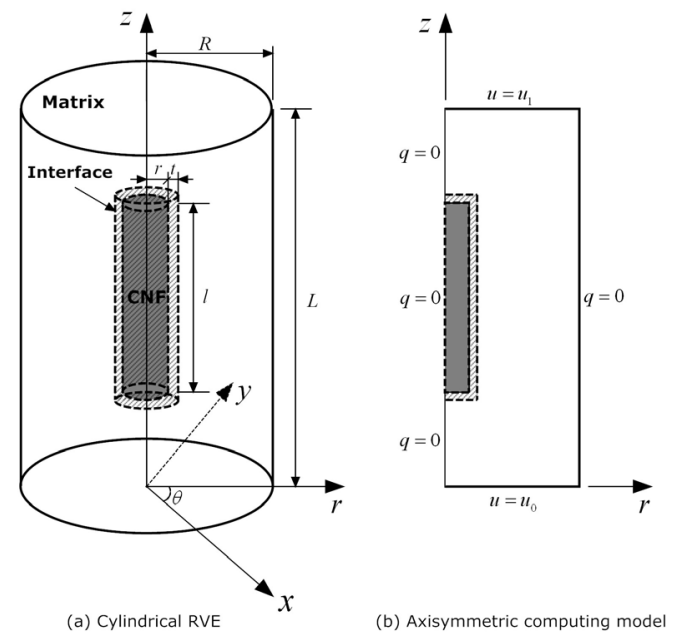

Figure 1: Nanoscale axisymmetric cylindrical RVE containing single CNT.

According to Fourier's law, the thermal conductivity along the $z$-direction is defined as

$$
k_{z}=-\frac{q_{z}}{\partial u / \partial z}=-\frac{q_{z}}{\left(u_{1}-u_{0}\right) / l}=-\frac{q_{z} l}{u_{1}-u_{0}} .
$$

which can be used to determine the effective thermal conductivity $k_{e}$ of the nanocomposite in the direction parallel to the fiber alignment by simply adjusting as

$$
k_{e}=-\frac{q_{z}^{\text {ave }} l}{u_{1}-u_{0}} .
$$


where $q_{z}^{\text {ave }}$ denotes the average values on the data-collection surfaces, i.e. the top and bottom surfaces of the cylindrical RVE. In Eq. (6), the fact that while the temperature varies linearly along the $z$-direction, then the heat flux component $q_{z}$ remains constant, has been used.

\subsection{FS for axisymmetric model}

The FS is critically important for implementation of the proposed algorithm. If the medium is isotropic, that is $k=k_{r}=k_{z}$, the FS for axisymmetric problems is usually derived by integrating the FS of three-dimensional steady-state heat conduction along the circumstance direction [13] and setting the source point $\mathbf{x}_{s}=\left(r_{s}, z_{s}\right)$ on the $O x z$ plane. As a result, we obtain

$$
u^{*}\left(\mathbf{x}, \mathbf{x}_{s}\right)=-\int_{0}^{2 \pi} \frac{1}{4 \pi \rho k} \mathrm{~d} \theta=-\frac{K(m)}{k \pi \sqrt{a+b}} .
$$

with

$$
m=\frac{2 b}{a+b}, a=r^{2}+r_{s}^{2}+\left(z-z_{s}\right)^{2}, \quad b=2 r r_{s}, \quad K(m)=\int_{0}^{\pi / 2} \frac{\mathrm{d} t}{\sqrt{1-m \sin ^{2} t}} .
$$

where $\mathbf{x}=(r, z)$ is a field point to be considered, $\rho$ is the distance between the field point and source point, and $k(m)$ represents the complete elliptic integral of the first kind.

\section{FS based FE formulation}

In this section, a hybrid FE model with FS as an intra-element trial function is developed for the boundary value problem (BVP) defined by Eqs. (3) and (4).

\subsection{Non-conforming intra-element field}

Motivated by the idea of the method of FS (MFS) [14] to remove the singularity of the FS, for a particular element $e$ occupying sub-domain $\Omega_{e}$, the intra-element temperature field is extracted from a linear combination of FS, that is,

$$
u_{e}(\mathbf{x})=\sum_{j=1}^{n_{s}} G\left(\mathbf{x}, \mathbf{x}_{s j}\right) c_{e j}=\mathbf{N}_{e}(\mathbf{x}) \mathbf{c}_{e}, \quad \forall \mathbf{x} \in \Omega_{e}, \mathbf{x}_{s j} \notin \Omega_{e} .
$$

where $c_{e j}$ is undetermined coefficients and $n_{s}$ is the number of virtual sources outside the element $e$, which can be generated by the formulation in literatures [10-12].

To guarantee the requirement of $r r_{s} \geq 0$ in the evaluation of the elliptic integrals of the first and second kinds, the local coordinate system $(\breve{r}, \breve{z})$ must be employed, whose origin can be determined by means of the minimum values of the coordinates of source points. 
The corresponding normal heat flux on $\Gamma_{e}$ is given by

$$
q_{e}=\left[\begin{array}{ll}
n_{r} & n_{z}
\end{array}\right]\left[\begin{array}{l}
q_{r} \\
q_{z}
\end{array}\right]=\mathbf{Q}_{e} \mathbf{c}_{e} .
$$

where

$$
\mathbf{Q}_{e}=-k\left[\begin{array}{ll}
n_{r} & n_{z}
\end{array}\right]\left[\begin{array}{l}
\mathbf{N}_{e, r} \\
\mathbf{N}_{e, z}
\end{array}\right] .
$$

\subsection{Auxiliary conforming frame field}

In order to enforce conformity on the field variable $u$, for instance, $u_{e}=u_{f}$ on $\Gamma_{e} \cap \Gamma_{f}$ of any two neighboring elements $e$ and $f$, an auxiliary inter-element frame field $\tilde{u}$ is used and expressed in terms of the same degrees of freedom (DOF), $\mathbf{d}_{e}$, as used in conventional FEM

$$
\tilde{u}_{e}(\mathbf{x})=\tilde{\mathbf{N}}_{e}(\mathbf{x}) \mathbf{d}_{e}, \quad \mathbf{x} \in \Gamma_{e} .
$$

where $\tilde{\mathbf{N}}_{e}$ represents the conventional shape functions used in conventional FEM.

\subsection{Modified variational principle and stiffness equation}

For the BVP defined in Eqs. (3) and (4), since the stationary conditions of the traditional potential or complementary variational functional cannot guarantee satisfaction of the inter-element continuity condition required in the proposed HFS FE model, a modified potential functional is developed as follows:

$$
\frac{\Pi_{m e}}{\pi}=-\int_{A_{e}}\left\{k_{r}\left(\frac{\partial u}{\partial r}\right)^{2}+k_{z}\left(\frac{\partial u}{\partial z}\right)^{2}-2 G u\right\} r \mathrm{~d} A-\int_{\Gamma_{q e}} \bar{q} \tilde{u} r \mathrm{~d} \Gamma+\int_{\Gamma_{e}}(\tilde{u}-u) q r \mathrm{~d} \Gamma \text {. }
$$

in which the governing equation (3) is assumed to be satisfied, a priori, due to the use of FS as intra-element trial functions.

Having independently defined the intra-element field and frame field in a particular element $e$, the next step is to generate the element stiffness equation by substituting those fields into the proposed functional (13).

Due to the constant coefficient $\pi$ appearing in Eq. (13) having no effect on the final result, it is discarded. As a result, the variational functional $\Pi_{m e}$ without the heat generation $G$ can be rewritten as

$$
\Pi_{m e}=-\frac{1}{2} \int_{A_{e}}\left\{k_{r}\left(\frac{\partial u}{\partial r}\right)^{2}+k_{z}\left(\frac{\partial u}{\partial z}\right)^{2}\right\} r \mathrm{~d} A-\int_{\Gamma_{q e}} \bar{q} \tilde{u} r \mathrm{~d} \Gamma+\int_{\Gamma_{e}}(\tilde{u}-u) q r \mathrm{~d} \Gamma .
$$

Applying the Gaussian theorem to the element functional (14), we finally have the following functional defined on the element boundary

$$
\Pi_{m e}=-\frac{1}{2} \int_{\Gamma_{e}} q u r \mathrm{~d} \Gamma-\int_{\Gamma_{q e}} \bar{q} \tilde{u} \mathrm{~d} \Gamma+\int_{\Gamma_{e}} q \tilde{u} r \mathrm{~d} \Gamma .
$$


which, by substituting Eqs. (9), (10) and (12) into the functional (15), yields

$$
\Pi_{e}=-\frac{1}{2} \mathbf{c}_{e}^{\mathrm{T}} \mathbf{H}_{e} \mathbf{c}_{e}-\mathbf{d}_{e}^{\mathrm{T}} \mathbf{g}_{e}+\mathbf{c}_{e}^{\mathrm{T}} \mathbf{G}_{e} \mathbf{d}_{e} .
$$

with

$$
\mathbf{H}_{e}=\int_{\Gamma_{e}} \mathbf{Q}_{e}^{\mathrm{T}} \mathbf{N}_{e} r \mathrm{~d} \Gamma, \quad \mathbf{G}_{e}=\int_{\Gamma_{e}} \mathbf{Q}_{e}^{\mathrm{T}} \tilde{\mathbf{N}}_{e} r \mathrm{~d} \Gamma, \quad \mathbf{g}_{e}=\int_{\Gamma_{e q}} \tilde{\mathbf{N}}_{e}^{\mathrm{T}} \bar{q} r \mathrm{~d} \Gamma .
$$

Minimization of the functional $\Pi_{e}$ with respect to $\mathbf{c}_{e}$ and $\mathbf{d}_{e}$, respectively, gives

$$
\frac{\partial \Pi_{e}}{\partial \mathbf{c}_{e}^{\mathrm{T}}}=-\mathbf{H}_{e} \mathbf{c}_{e}+\mathbf{G}_{e} \mathbf{d}_{e}=\mathbf{0}, \quad \frac{\partial \Pi_{e}}{\partial \mathbf{d}_{e}^{\mathrm{T}}}=\mathbf{G}_{e}^{\mathrm{T}} \mathbf{c}_{e}-\mathbf{g}_{e}=\mathbf{0} .
$$

from which the optional relationship between $\mathbf{c}_{e}$ and $\mathbf{d}_{e}$, and the stiffness equation, can be produced

$$
\mathbf{K}_{e} \mathbf{d}_{e}=\mathbf{g}_{e}, \quad \mathbf{c}_{e}=\mathbf{H}_{e}^{-1} \mathbf{G}_{e} \mathbf{d}_{e} .
$$

where $\mathbf{K}_{e}=\mathbf{G}_{e}^{\mathrm{T}} \mathbf{H}_{e}^{-1} \mathbf{G}_{e}$ stands for the element stiffness matrix.

Assembling the element stiffness matrix $\mathbf{K}_{e}$ and the right-hand vector $\mathbf{g}_{e}$ gives the following set of equations

$$
\mathbf{K d}=\mathbf{g} .
$$

where $\mathbf{K}, \mathbf{d}$ and $\mathbf{g}$ are global quantities.

\section{Numerical results and discussion}

In this section, the proposed axisymmetric hybrid FE model for the thermal analysis of the cylindrical RVE is first validated by comparing the results obtained to those from ABAQUS. Then, the hybrid model is applied to study the effects of nanofiber and the interphase on the effective thermal conductivity of the resulting composites. The material elastomer S160, having a thermal conductivity $0.56 \mathrm{~W} / \mathrm{mK}$, is chosen as matrix, while the nanofiber is assumed to have a thermal conductivity $1000 \mathrm{~W} / \mathrm{mK}$. The thermal property of the interphase is assigned over the interval $[0.01 \sim 1] \times 0.56 \mathrm{~W} / \mathrm{mK}$. Additionally, for the sake of convenience, the remaining data used in the analysis is tabulated in Table 1.

In the calculation, a total of 160 eight-node elements with 569 nodes are used to model the computing domain for different cases, i.e. $t_{i}=1.25 \mathrm{~nm}$ and $t_{i}=2.5 \mathrm{~nm}$, illustrated in Fig. 2, in which the red region represents the interface elements, the yellow region denotes the nanofiber elements, and the white region represents the matrix elements. The computing codes are written in MATLAB and used to evaluate the HFS-FEM results of the problem. 
Table 1: $\quad$ Geometry and boundary conditions for cylindrical RVE.

\begin{tabular}{c|c}
\hline Parameters & Values \\
\hline RVE length $L$ & $100 \mathrm{~nm}$ \\
RVE radius $R_{0}$ & $10 \mathrm{~nm}$ \\
Nanofiber length $l$ & $25 \mathrm{~nm}, 50 \mathrm{~nm}, 75 \mathrm{~nm}, 100 \mathrm{~nm}$ \\
Nanofiber radius $r_{0}$ & $2.5 \mathrm{~nm}, 5 \mathrm{~nm}, 7.5 \mathrm{~nm}$ \\
Interface thickness $t_{i}$ & $1.25 \mathrm{~nm}, 2.5 \mathrm{~nm}$ \\
Temperature at the upper surface $u_{1}$ & $100 \mathrm{~K}$ \\
Temperature at the lower surface $u_{0}$ & $200 \mathrm{~K}$ \\
\hline
\end{tabular}

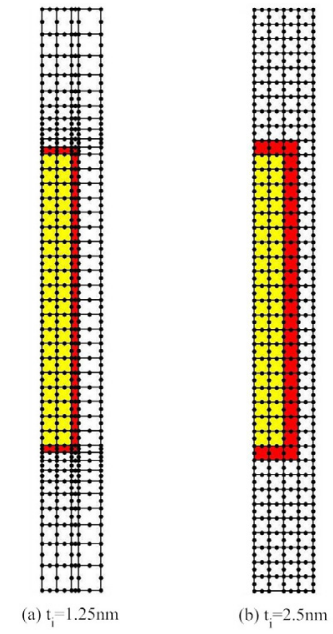

Figure 2: $\quad$ Two mesh divisions used for HFS-FEM.

\subsection{Validation of the proposed approach}

To validate the proposed algorithm, the temperature distributions in the RVE considered are calculated and compared with those from ABAQUS, in the absence of an interfacial layer. Since the thermal conductivity of the filler is about 1786 times higher than that of the matrix, this difference is sufficient to permit most of the heat flux to pass through the fiber portion of the composites. To examine the thermal effect in the cylindrical system, the length and radius of the fiber are assumed to be $50 \mathrm{~nm}$ and $5 \mathrm{~nm}$, respectively. Fig. 3 plots the temperature distribution along the line $r=0,5,7.5,10 \mathrm{~nm}$, and all results are compared to those from ABAQUS with the same meshes. As expected, there is a reasonably good agreement between them. Moreover, the temperature distribution for the case without the filler is also provided in Fig. 3 to demonstrate the effect of the filler. It can be clearly seen that the presence of the 
filler assists in reducing the overall temperature in the lower region $(0 \leq 2 z \leq L-l)$, whereas the overall temperature in the upper region, that is $L+l \leq 2 z \leq 2 L$, increases, compared to the corresponding temperature without fiber. Simultaneously, we also find that the temperature in the fiber is almost constant (about 149.8K). Moreover, near the tip of the fiber, the temperature changes at a more rapid rate than the surface temperature of the filler.

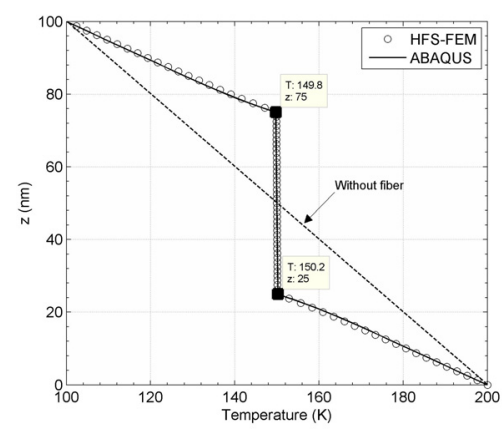

(a) $r=0 \mathrm{~nm}$

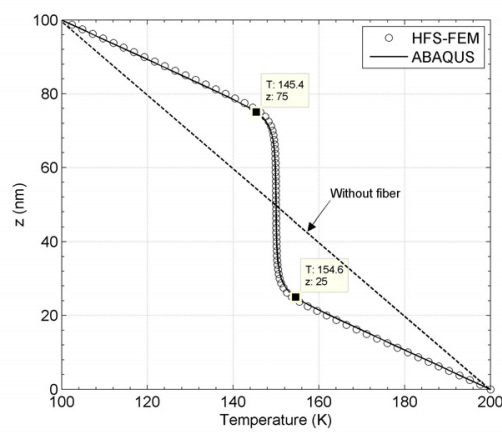

(c) $r=7.5 \mathrm{~nm}$

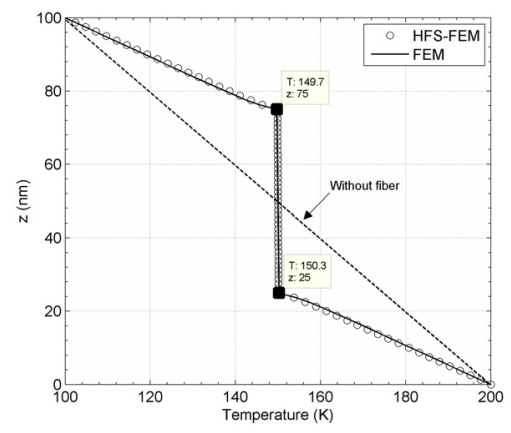

(b) $r=5 \mathrm{~nm}$

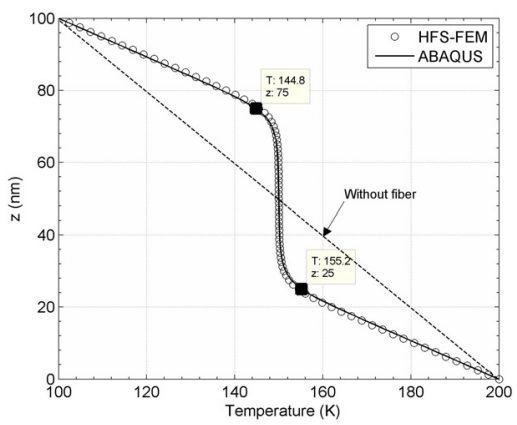

(d) $r=10 \mathrm{~nm}$

Figure 3: Temperature distribution with $l=50 \mathrm{~nm}$ and $r_{0}=5 \mathrm{~nm}$.

\subsection{Effect of nanofiber size on thermal properties of the composites}

To investigate the size effect of the nanofiber on the overall thermal properties of the nanocomposite in the absence of an interfacial layer, two different cases are considered. In the first case the length of the nanofiber is kept constant and its radius changes; for example, we take $l=50 \mathrm{~nm}$ and $r_{0}=2.5 \mathrm{~nm}, 5.0 \mathrm{~nm}$ and $7.5 \mathrm{~nm}$. The results given in Fig. 4 show that an increase in the radius of the fiber induces a larger value of the effective thermal conductivity $k_{e}$ of the composites. However, this effect is not very significant as the effective thermal property of the nanocomposite changes only slightly even when the radius is increased from $2.5 \mathrm{~nm}$ to $7.5 \mathrm{~nm}$. 


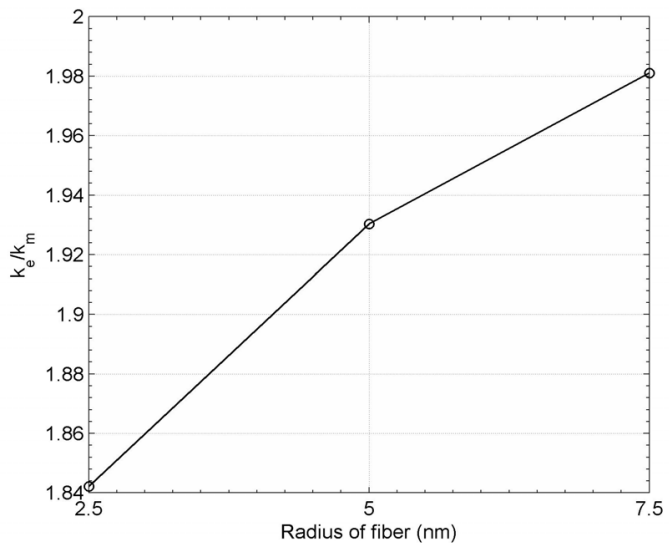

Figure 4: Effect of nanofiber radius on the effective thermal conductivity of nanocomposite with constant length $l=50 \mathrm{~nm}$.

In contrast, the length of the fiber has a more significant effect on the effective thermal conductivity $k_{e}$. The results in Fig. 5 show that for a given radius $r_{0}=5 \mathrm{~nm}$, when the length $l$ of the filler increases from $25 \mathrm{~nm}$ to $100 \mathrm{~nm}$, that is, the filler extends through the matrix, the ratio $k_{e} / k_{m}$ rapidly increases from 1.3 to 14.5 .

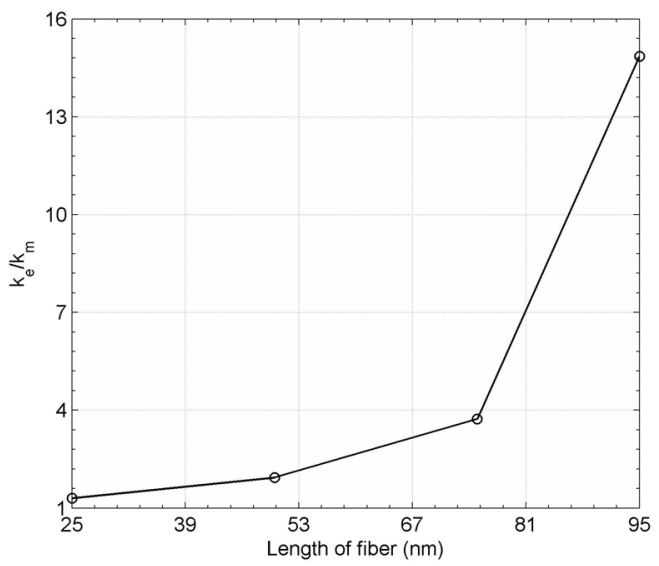

Figure 5: Effect of nanofiber length on the effective thermal conductivity of nanocomposite with constant radius $r_{0}=5 \mathrm{~nm}$.

\subsection{Effect of the interphase on effective thermal conduction}

To investigate the interface effect on the thermal conductivity of the nanocomposites, various values of interface conductivity and thickness are 
considered. In the computation, the length and radius of the fiber remain invariant at $50 \mathrm{~nm}$ and $5 \mathrm{~nm}$, respectively, while the thermal conductivity of the interphase changes in the range $[0.01,1] \times 0.56 \mathrm{~W} / \mathrm{mK}$, making the thickness of the interphase $1.25 \mathrm{~nm}$ and $2.5 \mathrm{~nm}$, respectively. Fig. 6 shows the variation of effective thermal conductivity $k_{e}$ of the composites by considering the existence of interphase. It can be seen that the interface thickness has an insignificant effect on the effective property of the composite. Furthermore, variation of the interface property has a significant effect on the effective conductivity of the composite, and a decrease in the value of interface conductivity induces a decrease in the overall conductivity of the composite.

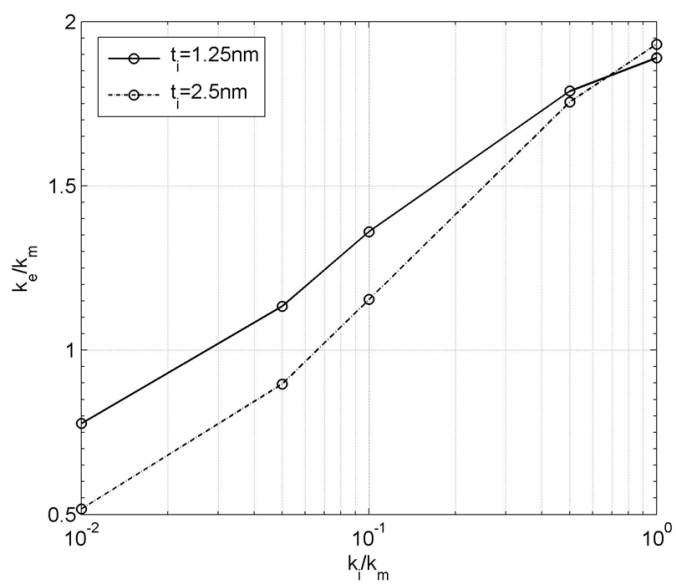

Figure 6: Effect of interface thickness and conductivity on the effective thermal conductivity of the composite.

\section{Conclusion}

In this paper an axisymmetric model of heat conduction in a cylindrical RVE is developed and used to study the effective thermal properties of nanocomposites. The proposed hybrid FE formulation involves element boundary integrals only, by virtue of use of the FS of the problem as an intra-element trial function. Using the proposed model, the effective thermal conductivity is calculated for various model parameters, including various sizes of nanofiller, and various thickness and material parameters of the interface. The numerical results show that the surface temperature of the nanofiller remains almost constant during heat transfer, and the overall thermal properties of the nanocomposites are affected largely by the length of the nanofiller and the material parameters of the interface, but only slightly affected by the radius of the nanofiller and the interfacial thickness. 


\section{References}

[1] Moniruzzaman, M., Sahin, A. \& Winey, K.I., Improved mechanical strength and electrical conductivity of organogels containing carbon nanotubes. Carbon, 47, pp. 645-650, 2009.

[2] Agarwal, S., Khan, M.M.K. \& Gupta, R.K., Thermal conductivity of polymer nanocomposites made with carbon nanofibers. Polymer Engineering and Science, 48, pp. 2474-2481, 2008.

[3] Clancy, T.C. \& Gates, T.S., Modeling of interfacial modification effects on thermal conductivity of carbon nanotube composites. Polymer, 47, pp. 5990-5996, 2006.

[4] Liu, Y.J. \& Chen, X.L., Continuum models of carbon nanotube-based composites using the boundary element method. Electronic Journal of Boundary Elements, 1, pp. 316-335, 2003.

[5] Nishimura, N. \& Liu, Y.J., Thermal analysis of carbon-nanotube composites using a rigid-line inclusion model by the boundary integral equation method. Computational Mechanics, 35, pp. 1-10, 2004.

[6] Zhang, J.M., Tanaka, M., Matsumoto, T. \& Guzik, A., Heat conduction analysis in bodies containing thin-walled structures by means of hybrid BNM with an application to CNT-based composites. JSME-Series A, 47, pp. 181-188, 2004.

[7] Zhang, J.M., Tanaka, M. \& Matsumoto, T., A simplified approach for heat conduction analysis of CNT-based nano-composites. Computer Methods in Applied Mechanics and Engineering, 193, pp. 5597-5609, 2004.

[8] Singh, I.V., Tanaka, M. \& Endo, M., Thermal analysis of CNT-based nano-composites by element free Galerkin method. Computational Mechanics, 39, pp. 719-728, 2007

[9] Singh, I.V., Tanaka, M. \& Endo, M., Effect of interface on the thermal conductivity of carbon nanotube composites. International Journal of Thermal Sciences, 46, pp. 842-847, 2007.

[10] Wang, H. \& Qin, Q.H., Hybrid FEM with Fundamental Solutions as trial functions for Heat Conduction Simulation. Acta Mechanica Solida Sinica, 22, pp. 487-498, 2009.

[11] Wang, H. \& Qin, Q.H., FE approach with Green's function as internal trial function for simulating bioheat transfer in the human eye. Archives of Mechanics, 62, pp. 493-510, 2010.

[12] Wang, H. \& Qin, Q.H., Fundamental-solution-based finite element model for plane orthotropic elastic bodies. European Journal of Mechanics-A/Solids, 29, pp. 801-809, 2010.

[13] Brebbia, C.A. Telles, J.C.F. \& Wrobel, L.C., Boundary element techniques, Springer-Verlag: Berlin and New York, 1984.

[14] Wang, H. \& Qin, Q.H., A meshless method for generalized linear or nonlinear Poisson-type problems. Engineering Analysis with Boundary Elements, 30, pp. 515-521, 2006. 